PROCEEDINGS OF THE AMERICAN MATHEMATICAL SOCIETY

Volume 124, Number 11, November 1996

\title{
ON WEAK COMPACTNESS AND COUNTABLE WEAK COMPACTNESS IN FIXED POINT THEORY
}

\author{
JOHN KULESZA AND TECK-CHEONG LIM
}

(Communicated by Palle E. T. Jorgensen)

\begin{abstract}
We prove that weak compactness and countable weak compactness in metric spaces are not equivalent. However, if the metric space has normal structure, they are equivalent. It follows that some fixed point theorems proved recently are consequences of a classical theorem of Kirk.
\end{abstract}

Let $(X, d)$ be a metric space. Let $\mathcal{F}$ be the family of subsets of $X$ consisting of $X$ and sets which are complements of closed balls of $X$. The weak topology (also called ball topology) on $X$ is the topology whose open sets are generated by $\mathcal{F}$, i.e. $\mathcal{F}$ forms a subbase for the open sets of $X$. If $X$ is a subset of a Banach space, the weak topology defined in this sense is generally weaker than the usual weak topology on $X$. For details on this topology in a Banach space setting, see [1] and [2].

We say $X$ is weakly compact if it is compact in the weak topology. A subset of $X$ is called a ball-intersection if it is an intersection of closed balls. $X$ is said to have normal structure if every ball-intersection $D$ of $X$ containing more than one point contains a non-diametral point, i.e. a point $r \in D$ such that

$$
\sup \{d(r, x): x \in D\}<\operatorname{diam}(D) .
$$

It is clear that $X$ is weakly compact if and only if every nonempty family of ballintersections with the finite intersection property has a nonempty intersection. And by the Alexander subbase theorem, one can replace 'ball-intersections' with 'closed balls'. $X$ is countably weakly compact if every decreasing sequence of nonempty ball-intersections in $X$ has a nonempty intersection. Obviously every weakly compact metric space is countably weakly compact. In this paper we show that the converse is false. However, if the metric space has normal structure, the converse is true. This shows that the assumption of countable weak compactness in some theorems in the literature is not really weaker than that of weak compactness. It also shows that every complete metric space with uniform normal structure is weakly compact, thereby a fixed point theorem of Khamsi [3] actually follows from that of Kirk [4]. Throughout this paper, $B_{r}(p)$ will denote the closed ball centered at $p$ with radius $r$.

Received by the editors January 3, 1995 and, in revised form, March 27, 1995.

1991 Mathematics Subject Classification. Primary 47H10, 47H09; Secondary 54E50, 54D30.

Key words and phrases. Nonexpansive mappings, fixed point, normal structure, weak compact, countable weak compact, uniform normal structure. 
Example. This is an example of a countably weakly compact metric space that is not weakly compact. Let $\Omega$ be the first uncountable ordinal. Let $P$ be the set of ordinals $<\Omega$ and $Q$ be the set of countable subsets of $P$. Let $X=P \cup Q$ with the metric $d$ defined as follows:

$$
\begin{gathered}
d\left(p_{1}, p_{2}\right)=1.5 \quad \text { if } p_{1}, p_{2} \in P \text { and } p_{1} \neq p_{2}, \\
d\left(q_{1}, q_{2}\right)=1 \quad \text { if } q_{1}, q_{2} \in Q \text { and } q_{1} \neq q_{2}, \\
d(p, q)=d(q, p)=\left\{\begin{array}{ll}
1 & \text { if } p \in q \\
1.5 & \text { if } p \notin q
\end{array} \text { for } p \in P \text { and } q \in Q .\right.
\end{gathered}
$$

(We define without saying that $d(x, y)=0$ if $x=y$.) One checks that $d$ is indeed a metric.

We have the following six types of balls for $p \in P$ and $q \in Q$ :

$$
\begin{gathered}
B_{r}(p)=X \quad \text { if } r \geq 1.5, \\
B_{r}(p)=\{p\} \cup\{q: p \in q \in Q\} \quad \text { if } 1 \leq r<1.5, \\
B_{r}(p)=\{p\} \quad \text { if } 0 \leq r<1, \\
B_{r}(q)=X \quad \text { if } r \geq 1.5, \\
B_{r}(q)=\{p: p \in q, p \in P\} \cup Q \quad \text { if } 1 \leq r<1.5, \\
B_{r}(q)=\{q\} \quad \text { if } 0 \leq r<1 .
\end{gathered}
$$

To show that $X$ is not weakly compact, consider

$$
\mathcal{F}=\left\{B_{1}(p): p \in P\right\}
$$

If $\mathcal{F}_{1}=\left\{B_{1}\left(p_{n}\right): n=1,2, \ldots\right\} \subseteq \mathcal{F}$, then $q=\left\{p_{1}, p_{2}, \ldots\right\} \in \cap \mathcal{F}_{1}$ by (1b) above. So $\mathcal{F}$ has countable (and hence finite) intersection property. Let

$$
\mathcal{G}=\left\{B_{1}\left(p_{\alpha}\right): \alpha \in D\right\}, \quad D \text { uncountable, }
$$

be an uncountable subfamily of $\mathcal{F}$. Since every $q \in Q$ is countable, there exists $\alpha \in D$ such that $p_{\alpha} \notin q$ so $q \notin B_{1}\left(p_{\alpha}\right)$. Also, since $|D|>1$, for every $p \in P$ there exists $p_{\alpha} \neq p$ so $p \notin B_{1}\left(p_{\alpha}\right)$. This shows $\bigcap \mathcal{G}=\varnothing$ for every uncountable subfamily of $\mathcal{F}$. Thus, in particular, $\bigcap \mathcal{F}=\varnothing$. This proves that $X$ is not weakly compact.

To show that $X$ is countably weakly compact, first note from the six types of balls above that every ball-intersection containing more than one point must be either the whole $X$ or an intersection of countably many (otherwise it would be $\varnothing$ by the paragraph above) balls of type (1b) and some balls of type (2b). Thus such a ball-intersection $I$ is associated with a countable subset $C$ of $P(C=\varnothing$ if $I=X)$ such that $q \in I$ whenever $q \supseteq C$ and $q \in Q$. Now suppose $\mathcal{I}=\left\{I_{i}: i=1,2, \ldots\right\}$ is a decreasing sequence of nonempty ball-intersections in $X$. If some of $I_{i}$ is a singleton, then obviously the intersection will be the singleton. Otherwise, let $C_{i}$ be the countable set associated with $I_{i}$ and let $C=\bigcup C_{i}$. Then $C$ is countable and belongs to the intersection $\bigcap I_{i}$. This shows that $X$ is countably weakly compact.

We now proceed to prove the following result.

Theorem 1. Every countably weakly compact metric space with normal structure is weakly compact. 
Lemma 1. If $X$ is countably weakly compact but not weakly compact, then there exists an uncountable cardinal $K$ and a decreasing transfinite sequence $\left\{I_{\alpha}: \alpha<K\right\}$ of nonempty ball-intersections such that $\bigcap\left\{I_{\alpha}: \alpha<K\right\}=\varnothing$.

Proof. Since $X$ is not weakly compact, there exists a family $\mathcal{F}$ of nonempty ballintersections of $X$ with the finite intersection property and $\cap \mathcal{F}=\varnothing$. Let $K$ be the smallest cardinal such that there exists a subfamily $\mathcal{G}$ of $\mathcal{F}$ with cardinality $K$ and $\bigcap \mathcal{G}=\varnothing$. Since $X$ is countably weakly compact, $K$ must be uncountable. Well order $\mathcal{G}$ so $\mathcal{G}=\left\{B_{\gamma}: \gamma<K\right\}$. Now for every $\alpha<K$ define $I_{\alpha}=\bigcap\left\{B_{\gamma}: \gamma \leq \alpha\right\}$. By the minimality of $K$, each $I_{\alpha} \neq \varnothing$. Also $\bigcap\left\{I_{\alpha}: \alpha<K\right\}=\bigcap \mathcal{G}=\varnothing$.

We shall call a decreasing transfinite sequence as in Lemma 1 a $\varnothing$-chain. For each $I_{\alpha}$ in a $\varnothing$-chain $I$, let $d_{\alpha}=\operatorname{diam}\left(I_{\alpha}\right)$. Since $K$ is uncountable and $d_{\alpha}$ is nonincreasing, there exists $\beta<K$ such that $d_{\alpha}=d$ for all $\alpha$ with $\beta<\alpha<K$. We have $d>0$ for otherwise $\bigcap\left\{I_{\alpha}: \alpha<K\right\}$ would be a singleton and hence nonempty - a contradiction. We shall call the number $d$ the diameter of the $\varnothing$ chain and denote it by $\operatorname{diam}(I)$. We shall call a $\varnothing$-chain $J$ a refinement of another $\varnothing$-chain $I$ if $J_{\alpha} \subseteq I_{\alpha}$ for every $\alpha<K$.

Lemma 2. Under the hypothesis of Lemma 1, there exists a $\varnothing$-chain $I^{*}$ with a minimal diameter, i.e. if $J$ is a refinement of $I^{*}$, then $\operatorname{diam}(J)=\operatorname{diam}\left(I^{*}\right)$.

Proof. Let $I$ be a $\varnothing$-chain as in Lemma 1 . Define a sequence of $\varnothing$-chains $I_{j}$, $j=0,1,2, \ldots$, inductively as follows. Let $I_{0}=I$. Suppose $I_{j}$ has been defined. Let $\hat{d}_{j}=\inf \left\{\delta\right.$ : there exists a refinement of $I_{j}$ with diameter $\left.\delta\right\}$. Then define $I_{j+1}$ to be a refinement of $I_{j}$ with $0 \leq \operatorname{diam}\left(I_{j+1}\right)-\hat{d}_{j}<\frac{1}{j+1}$. For each $\alpha<K$ define $I_{\alpha}^{*}=\bigcap\left\{I_{j \alpha}: j=0,1, \ldots\right\}$. By countable weak compactness, each $I_{\alpha}^{*}$ is nonempty. Obviously $I^{*}$ is a $\varnothing$-chain. Suppose $J$ is a refinement of $I^{*}$. For every $j$, since both $J$ and $I^{*}$ are refinements of $I_{j+1}$, we have $\left|\operatorname{diam}(J)-\operatorname{diam}\left(I^{*}\right)\right| \leq \operatorname{diam}\left(I_{j+1}\right)-\hat{d}_{j}<$ $\frac{1}{j+1}$. This proves that $\operatorname{diam}(J)=\operatorname{diam}\left(I^{*}\right)$.

Proof of Theorem 1. Suppose $X$ is countably weakly compact and has normal structure. Suppose on the contrary $X$ is not weakly compact. Then $X$ contains a $\varnothing$ chain $I=\left\{I_{\alpha}: \alpha<K\right\}$ with a minimal diameter as in Lemma 2. Since $K$ is an uncountable cardinal, there exists $\beta<K$ such that $\operatorname{diam}\left(I_{\alpha}\right)=\operatorname{diam}(I)=d$ for all $K>\alpha>\beta$. We have $d>0$ for otherwise the intersection of $I$ would be a singleton. By normal structure, for each $\beta<\alpha<K$ there exists $0<r_{\alpha}<\operatorname{diam}\left(I_{\alpha}\right)=d$ and $p_{\alpha} \in I_{\alpha}$ such that $I_{\alpha} \subseteq B_{r_{\alpha}}\left(p_{\alpha}\right)$. Let $S_{n}=\left[d\left(1-\frac{1}{n}\right), d\left(1-\frac{1}{n+1}\right)\right), n=1,2, \ldots$ Let $F_{n}=\left\{\alpha: \beta<\alpha<K, r_{\alpha} \in S_{n}\right\}$. Since $K$ is an uncountable cardinal, $F_{m}$ must be cofinal in $K$ for some $m$. Let $r=d\left(1-\frac{1}{m+1}\right)$. Let $J=\bigcap\left\{B_{r}\left(p_{\alpha}\right): \alpha \in F_{m}\right\}$. One easily checks that $\left\{p_{\alpha}: \alpha \in F_{m}\right\} \subseteq J$. Let $M=\bigcap\left\{B_{r}(x): x \in J\right\}$. Since $\left\{p_{\alpha}: \alpha \in F_{m}\right\} \subseteq J$, we have $M \subseteq J$. Suppose $y, z \in M$. Then $z \in J$ and $y \in B_{r}(z)$, so $d(y, z) \leq r$. This implies $\operatorname{diam}(M) \leq r$. Also, $x \in J$ and $\alpha \in F_{m} \Rightarrow x \in B_{r}\left(p_{\alpha}\right) \Rightarrow p_{\alpha} \in B_{r}(x)$. So $\left\{p_{\alpha}: \alpha \in F_{m}\right\} \subseteq M$. Now define $I^{*}$ by setting $I_{\alpha}^{*}=I_{\alpha}$ for $\alpha \leq \beta$ and $I_{\alpha}^{*}=I_{\alpha} \cap M$ for $\beta<\alpha<K$. Note that $\left\{p_{\gamma}: \gamma \in F_{m}, \gamma \geq \alpha\right\} \subseteq I_{\alpha}^{*}$ for $\beta<\alpha<K$ and so $I_{\alpha}^{*} \neq \varnothing$. Since $\operatorname{diam}\left(I^{*}\right) \leq \operatorname{diam}(M)<d$, we have a contradiction to the minimality of $I$.

The metric version of Kirk's fixed point theorem [4] is the following:

Theorem 2. Let $X$ be a bounded weakly compact metric space with normal structure. Then every nonexpansive mapping $T: X \rightarrow X$ has a fixed point. 
It follows from Theorem 1 that a countable version of a fixed point theorem of Kirk [5], [6] actually follows from Theorem 2 above.

A metric space is said to have uniform normal structure if there exists a number $0<c<1$ such that for every ball-intersection $B$ of $X$ with $\operatorname{diam}(B)>0$, there is a point $z$ in $B$ with

$$
\sup \{d(z, x): x \in B\} \leq c \operatorname{diam}(B) .
$$

Khamski [3] proved that every complete metric space with uniform normal structure is countably weakly compact. His proof made use of a fixed point theorem which we state as a corollary below. We give here an alternative proof which enables us to conclude the fixed point theorem as a corollary.

Let $\mathcal{A}=\left\{A_{n}: n=1,2, \ldots\right\}$ and $\mathcal{B}=\left\{B_{n}: n=1,2, \ldots\right\}$ be two sequences of sets. We say that $\mathcal{A} \leq \mathcal{B}$ if $A_{n} \subseteq B_{n}$ for every $n$. If $\mathcal{K}=\left\{K_{n}: n=1,2, \ldots\right\}$ is a decreasing sequence of ball-intersections in a metric space and $\mathcal{C}=\left\{C_{n}: n=\right.$ $1,2, \ldots\}$ a sequence $\leq \mathcal{K}$, then by taking intersection, one can easily see that there is a smallest (relative to $\leq$ ) decreasing sequence of ball-intersections $\geq \mathcal{C}$. For a subset $B$ of a metric space, we shall denote by $\operatorname{Ad}(B)$ the smallest ball-intersection containing $B$.

Theorem 3. Every complete metric space with uniform normal structure is weakly compact.

Proof. Suppose $X$ is a complete metric space with uniform normal structure and let $c$ be the constant as in the definition. By Theorem 1, it suffices to prove that $X$ is countably weakly compact. Let $K_{n}, n=1,2, \ldots$, be a decreasing sequence of nonempty ball-intersections. Let $d=c \operatorname{diam}\left(K_{1}\right)$. For each $n$, let $F_{n}=\left\{x \in K_{n}: \sup _{y \in K_{n}} d(x, y) \leq d\right\}$. Each $F_{n}$ is a nonempty ball-intersection and has a diameter $\leq d$. Let $B_{n}, n=1,2, \ldots$, be the smallest (relative to the order $\leq$ ) decreasing sequence of ball-intersections containing $F_{n}, n=1,2, \ldots$ By the minimality, we have $B_{1}=\operatorname{Ad}\left(B_{2} \cup F_{1}\right), B_{2}=\operatorname{Ad}\left(B_{3} \cup F_{2}\right), \ldots$ Let $C_{n}=\left\{x \in B_{n}\right.$ : $\left.\sup _{y \in B_{n}} d(x, y) \leq d\right\}$. If $x \in C_{2}$, then $B_{d}(x) \supseteq B_{2}$. Since $x \in C_{2} \subseteq B_{2} \subseteq K_{2} \subseteq K_{1}$, we also have $B_{d}(x) \supseteq F_{1}$. It follows that $B_{d}(x) \supseteq \operatorname{Ad}\left(B_{2} \cup F_{1}\right)=B_{1}$ and hence $x \in C_{1}$. This shows $C_{2} \subseteq C_{1}$. Similarly, one shows that $C_{n}, n=1,2, \ldots$, is decreasing. It is easy to see that $\operatorname{diam}\left(C_{n}\right) \leq d=c \operatorname{diam}\left(K_{1}\right)$. Repeating the process to $C_{2}, C_{3}, \ldots$, we obtain a decreasing sequence $D_{2}, D_{3}, \ldots$ such that $D_{n} \subseteq C_{n}$ and $\operatorname{diam}\left(D_{n}\right) \leq c^{2} \operatorname{diam}\left(K_{1}\right)$. Continuing in this manner, we see that there exists a decreasing sequence $\left\{L_{n}: n=1,2, \ldots\right\}$ of ball-intersections $\leq \mathcal{K}$ with $\operatorname{diam}\left(L_{n}\right) \leq c^{n} \operatorname{diam}\left(K_{1}\right)$. By the completeness of $X, \cap L_{n}$ is a singleton contained in $\bigcap K_{n}$ and hence $\bigcap K_{n} \neq \varnothing$.

The following corollary follows immediately from Theorems 2 and 3.

Corollary 1 (Khamsi [3]). Every bounded complete metric space with uniform normal structure has the fixed point property for nonexpansive mappings.

\section{REFERENCES}

1. H. H. Corson and J. Lindenstrauss, On weakly compact subsets of Banach spaces, Proc. Amer. Math. Soc. 17 (1966), 407-412. MR 33:7812

2. G. Godefroy and N. Kalton, The ball topology and its applications, Banach Space Theory (B. L. Lin, ed.), Contemp. Math., vol. 85, Amer. Math. Soc., Providence, RI, 1989, pp. 195237. MR 90c: 46022 
3. M. A. Khamsi, On metric spaces with uniform normal structure, Proc. Amer. Math. Soc. 106 (1989), 723-726. MR 90c:54028

4. W. A. Kirk, A fixed point theorem for mappings which do not increase distances, Amer. Math. Monthly 72 (1965), 1004-1006. MR 32:6436

5. __ Nonexpansive mappings in metric and Banach spaces, Estratto Dai, Rend. Sem. Mat. Fis. Milano, 51 (1981), 133-144. MR 84j:47091

6. __ An abstract fixed point theorem for nonexpansive mappings, Proc. Amer. Math. Soc. 82 (1981), 640-642. MR 82f:54075

Department of Mathematics, George Mason University, 4400 University Drive, FairFAX, VIRGINIA 22030

E-mail address: jkulesza@gmu.edu

E-mail address: tlim@gmu.edu 